\title{
Deploying Edge Computing Nodes for Large-scale IoT: A Diversity Aware Approach
}

\author{
Zhiwei Zhao, Member, IEEE, Geyong Min*, Member, IEEE, Weifeng Gao, Student Member, IEEE, \\ Yulei Wu, Member, IEEE, Hancong Duan, Member, IEEE, and Qiang Ni, Member, IEEE
}

\begin{abstract}
The recent advances in microelectronics and communications have led to the development of large-scale IoT networks, where tremendous sensory data is generated and needs to be processed. To support real-time processing for large-scale IoT, deploying edge servers with storage and computational capability is a promising approach. In this paper, we carefully analyze the impacting factors and key challenges for edge node deployment. We then propose a novel three-phase deployment approach which considers both traffic diversity and the wireless diversity of IoT. The proposed work aims at providing real-time processing service for the IoT network and reducing the required number of edge nodes. We conducted extensive simulation experiments, the results show that compared to the existing works that overlooked the two kinds of diversities, the proposed work greatly reduces the number of edge nodes and improves the throughput between IoT and edge nodes.
\end{abstract}

Index Terms-IoT, Large-scale, Edge Computing, Deployment

\section{INTRODUCTION}

The recent advances in low-power wireless communications and computing technologies have enabled the largescale implementation of Internet of Things (IoT) systems [1], where massive sensors, micro-controllers and transceivers are embedded to the facilities of buildings, vehicles, wearable items and wild areas [2], [3], [4]. The IoT aims at making the Internet even more immersive and pervasive, providing interactive cyber-physical access and control services [5], [6]. Based on the IoT infrastructure, various large-scale real-time applications emerge, which makes the real-time processing a fundamental and critical service for IoT [7], [8]. For example, the smart building system [4] consists various types of IoT sensory nodes including HD cameras, wearable sensors, localization anchor sensors, gym equipment sensors, etc. Those sensors need to keep collecting the sensory data continuously and provide real-time response to the upper level applications. For example, the health monitoring system needs to collect various health data from the wearable sensors from users and alarm when abnormal phenomenon is detected.

The large amount of sensory data and the corresponding real-time processing workload pose significant challenges to

Z. Zhao, W. Gao and Hancong Duan are with School of Computer Science and Engineering, University of Electronic Science and Technology of China, Chengdu, China. G. Min and Y. Wu are with University of Exeter, Exeter, UK. Q. Ni is with Lancaster University, Lancaster, UK.

G. Min is the corresponding author.

Copyright (c) 2012 IEEE. Personal use of this material is permitted. However, permission to use this material for any other purposes must be obtained from the IEEE by sending a request to pubs-permissions@ieee.org. the practical application of large-scale IoT systems. Meanwhile, edge computing [9] emerges as a promising solution to the above challenge as it provides instant storage and data processing services to the resource-constrained IoT nodes. Figure 1 shows the edge architecture for IoT systems. Multiple edge computing servers are deployed to cover part of the IoT nodes. The computational tasks and sensory data from the IoT nodes are sent to the edge nodes (ENs) for processing. The results are then returned to the IoT nodes or transmitted to the cloud for big data analytics [10].

Deployment of the edge nodes is a fundamental problem for the above architecture. Different from the existing works on sink deployment in multi-sink sensor networks, the deployment of edge nodes have several distinct challenges. First, compared to traditional sensor network nodes, IoT nodes are more diverse and have largely different traffic demands. For example, the video cameras produce much more data than the equipment maintenance sensor nodes. Second, unlike the mobile edge computing where $\mathrm{WiFi} /$ Cellular communications are utilized, IoT nodes often employ the low-power radios [11], [12] and are more prone to the wireless interference. Considering the edge servers are responsible for collecting data as well as disseminating data (e.g., for software update or computational feedback), the wireless interference can significantly affect the EN deployment.

To address the above challenges, we propose a deployment approach for edge servers in large-scale IoT. The proposed work has three building blocks. First, a traffic aware discretization approach is devised, which divides the whole IoT network area into a number of candidate positions. The discretization considers the traffic diversity of the IoT nodes and normalizes the demands/resources of the IoT nodes. Second, we propose a utility metric considering wireless diversity - link quality and link correlation to evaluate the candidate positions. Third, a deployment algorithm based on the utility metric is proposed. By incorporating both diversity information, the proposed work is expected to improve the throughput and reduce the required number of edge nodes. We conduct extensive simulation experiments and compare the proposed work to the existing works. The results show that our work achieves smaller number of edge nodes and greatly reduces the transmission count. The main contribution of this paper is summarized as follows.

1) We propose a discretization scheme to generate candidate positions, where traffic diversity is considered and the demands/resources of IoT nodes are normalized.

2) We propose a novel utility metric to evaluate the candidate positions, where the wireless diversity is consid- 


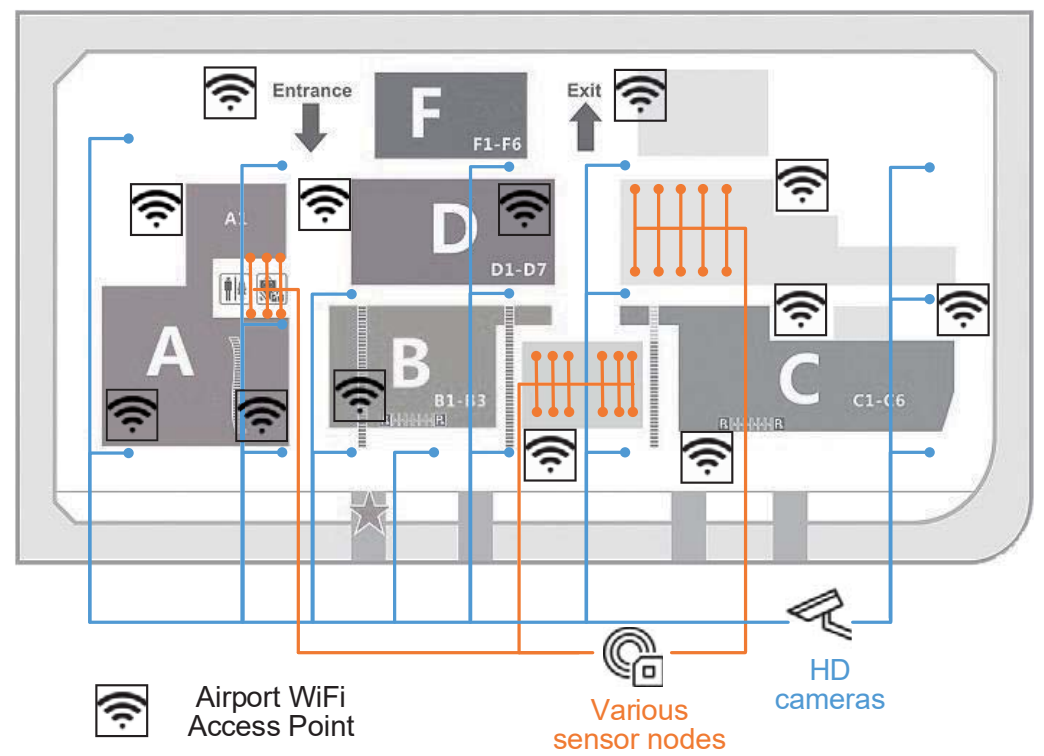

Fig. 1. An illustrative example for the system model. There are various types of IoT sensor nodes and wireless access points (AP) deployed in the target area. The edge nodes needs to cover the IoT nodes and try avoiding the interference from the wireless APs.

ered.

3) Based on the above schemes, we propose a deployment algorithm which improves the IoT-Edge throughput and reduces the number of edge nodes.

The remainder of the paper is organized as follow. Section II presents the preliminaries and system model for the edge deployment problem. Section III analyzes the challenges for the problem and presents the three-phase deployment approach. Section IV presents the simulation results and analysis. Section V presents the related works with edge computing and deployment in large-scale networks. Section VI concludes this paper and discusses future directions.

\section{Preliminaries and System Model}

Our aim is to deploy a number of edge nodes to a large-scale IoT network, where diverse IoT sensor nodes are in an area possibly with pedestrians and wireless interference. Figure 1 shows a typical IoT network in an airport, which consists a number of HD camera sensors with high traffic demands and a number of ordinary sensors with low traffic demands. The ordinary sensors are used for building monitoring, indoor navigation, equipment monitoring, etc. Although those IoT sensor nodes generates large amount of data, they usually have very limited computational resources for real-time data processing [7]. To support real-time data processing for the large-scale IoT network, a promising alternative is to deploy a number of edge nodes hierarchically with the IoT network, which are connected to the IoT nodes and processes the IoT data in real time. All IoT nodes then send the sensory data to the connected edge servers for data processing.

Compared to the traditional large-scale sensor network, the large-scale IoT network has two main differences.

1) First, the IoT networks are heterogeneous rather than homogeneous, which consists much more diverse IoT nodes. For example shown in the figure, the camera sensors and equipment sensors have largely different demands on data traffic and data processing.

2) Second, the IoT networks are often deployed in indoor environments rather than unmanned areas. Considering WiFi has been pervasively deployed for wireless access, the co-existence problem of edge nodes and the environmental wireless networks also needs to be considered. Specifically, as low power radios are often employed in the IoT nodes, they can be easily affected by WiFi communications, Bluetooth communiations, etc [13], [14].

Our goal now is to deploy a number of edge nodes to the IoT network to cover all the IoT nodes. The problem of minimizing the number of edge nodes is equivalent to the problem of Knapsack problem [15], which is NP Complete. Therefore in this paper, we design a heuristic to reduce the number of edge nodes and provide high-throughput data collection/dissemination service for real-time data processing in the IoT networks.

\section{Diversity Aware Deployment of the Edge SERVERS}

In this section, we present the three-phase deployment approach. Specially, we will present the details on considering traffic diversity and wireless diversity.

\section{A. Challenges}

There are two challenges to deploy edge nodes for realtime data processing in large-scale IoT - Traffic diversity and wireless diversity.

1) Traffic diversity. The IoT nodes are diverse in data types and traffic demands. Different types of data have 
different processing requirements and will require different amount of computational resources. Considering the edge nodes are often powerful, in this paper, we mainly consider the diversity of traffic demands. Different amount of traffic demands will directly affect the deployment of edge nodes in two ways. First, the edge nodes are targeted to receive data from the IoT nodes. Intuitively, the edge nodes should be deployed nearer to the IoT nodes with more traffic demands. Second, the traffic demands may not be consistent with the node density because different nodes have different demands. The two factors need to be jointly considered in the deployment process.

2) Wireless diversity. For wireless diversity we mainly consider link quality and link correlation. For many large-scale IoT systems, especially for those deployed in indoor environments, the WiFi networks, Bluetooth communications, even microwave ovens can have large impact on the transmission quality between the edge nodes and the IoT nodes as they typically use lowpower radios (e.g., ZigBee). Considering that edge nodes are used to collect sensory data and disseminate remote commands and maintenance instructions, both inbound and outbound performance can be largely impacted by the interference. Therefore, the link quality/correlation distribution and the impact on the performance of both collection/dissemination should also be considered in the deployment process.

\section{B. Overview}

To address the above challenges and deploy the edge nodes effectively, we incorporate the two kinds of diversities into the deployment process and propose a three-phase deployment approach. Figure 2 shows the overview of the proposed approach.

1) Discretization. Before determining the positions for deploying edge nodes, we first discretize the whole IoT network area into many small sections and the centroid of each section is a candidate position. In the discretization, we combine both wireless transmissions and the data traffic demands to define "effective" transmission levels, with which all nodes' levels are normalized and the traffic diversity is incorporated. The details are described in Section III-C.

2) A utility metric. Next, we propose a comprehensive metric to evaluate the impact of each candidate position. The utility metric calculates the expected performance gain of the candidate position regarding the expected number of transmissions required for both data collection and message dissemination. Wireless diversity including link quality and link correlation among multiple links are considered in the metric. The detailed design of the metric is described in Section III-D.

3) The deployment algorithm. Based on the proposed utility metric, we further devise a heuristic to select the best candidate positions for deploying the edge nodes. The input is the traffic demands and wireless

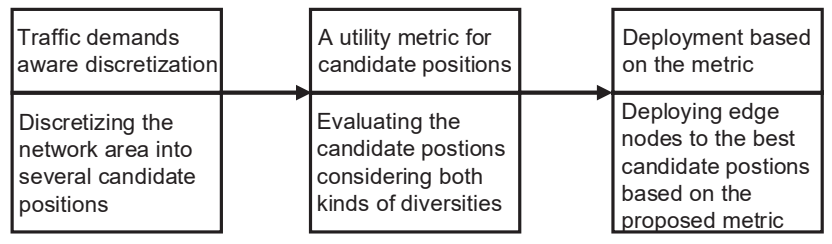

Fig. 2. Overview of the proposed work.

measurements (link quality/correlation) from the IoT network; The output is the positions for deploying edge nodes. We discussed the possible ways to reduce the measurement overhead in Section III-E.

The notations used throughout this paper is listed as follows.

TABLE I

THE NOTATIONS USED THROUGHOUT THIS PAPER.

\begin{tabular}{c|c}
\hline \hline Notations & Description \\
\hline$P_{c}$ & The set of all candidate positions. \\
\hline$P_{s}$ & The set of selected positions for deployment. \\
\hline$p_{i}$ & The $i$-th candidate position. \\
\hline$\rho_{i}$ & The proposed metric $\rho$ for candidate position $p_{i}$. \\
\hline$l_{i}^{n}$ & The distance of the $i$-th level of IoT node $n$. \\
\hline$d_{n}$ & The data traffic demand of node $n$. \\
\hline$t_{r}^{n}$ & The transmission rate from $n$ to position $r$. \\
\hline$u$ & The total number of levels considered in the discretization. \\
\hline$p_{k}^{c}$ & The collection performance for candidate position $p_{k}$. \\
\hline$p_{k}^{d}$ & The dissemination performance for candidate position $p_{k}$. \\
\hline$q_{i j}$ & The packet delivery ratio of link $i \rightarrow j$. \\
\hline$c_{i / j}^{n}$ & The link correlation between links $n \rightarrow i$ and $n \rightarrow j$. \\
\hline$c_{N_{i} / N_{j}}^{n}$ & The set link correlation between subset $N_{i}$ and $N_{j}$. \\
\hline \hline
\end{tabular}

\section{Discretization}

Given the positions of the IoT nodes, we can obtain a series of candidate positions for edge nodes deployment. In the existing works, the areas are divided by the levels of the communication quality. For example in Figure 3(a), the area is divided by the circles denoting the different transmission rates. Each block that lies in a combination of different levels of the IoT nodes is a candidate position. For each candidate position, the corresponding transmission rates are used for position selection. In the case shown in Figure 3(a), the optimal position for deploying en edge node is close to the three IoT sensors because it achieves the best average transmission rate.

However, while the transmission rate is a useful indicator for the sink nodes' effectiveness in homogeneous networks, it is no longer useful for heterogeneous networks. The reason is that different nodes have largely different traffic demands, leading to different task transfer delay. For example, the cameras have much more traffic loads than the ordinary sensors. The same transmission rate can lead to different task transfer delay for cameras and the ordinary sensors. Therefore, we redefine the levels for discretization, which incorporates the data 


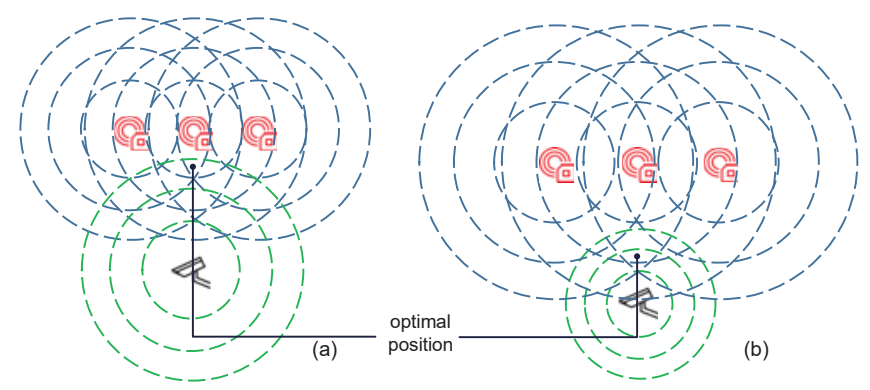

Fig. 3. The discretization approach with different leveling schemes. (a) shows the case that uses the transmission-rate levels for discretization; (b) shows the normalized levels which consider the data traffic demands from different IoT nodes.

traffic demands. The defined "normalized" level $i$ of node $n$ is calculated as

$$
l_{i}^{n}=\arg _{r}\left(\frac{t_{r}^{n}}{d_{n}}=1-\frac{i}{u}\right)
$$

where $i \in[0, u]$ denotes the $i$-th level and the percentage of the transmitted data out of the total transmission demand in unit time equals $1-\frac{i}{u} ; u$ denotes the total number of levels; $d_{n}$ denotes the transmission demand of node $n$ and $t_{r}^{n}$ denotes the expected transmission rate at the circle with radius $r$. For example, if we calculate ten levels for node $n$, then the second level of $n$ is $l_{0.8}^{n}$ which equals to the radius $r$ with which $\frac{t_{r}^{n}}{d_{n}}=0.8$. By such leveling scheme, the relationship between a node's effectiveness and the distance can be normalized. Figure 3(b) shows the normalized levels. We can see that compared to the original levels, the normalized levels for camera sensors become geographically smaller and the normalized levels for ordinary sensors become geographically larger. As a result, the optimal deployment position become closer to the camera. Since the cameras have high traffic demand and the ordinary sensors have low traffic demand, the new deployment can provide fast task offloading and real-time transmission for both nodes while the deployment in Figure 3(a) can provide real-time transmission for only the ordinary nodes.

It is also worth noting that the actual transmission rate at a given radius can be largely different due to the wireless interference. We will consider the impact of the wireless interference in the evaluation metric for the candidate positions in Section III-D. Once we have obtained the normalized levels for all IoT nodes, the target area has been divided into many candidate positions for EN deployment. We then use the utility metric to estimate the effectiveness of the candidate positions and then select the best deployment positions.

\section{A utility metric for candidate positions}

As the traffic diversity is considered in the discretization, now we need to take the wireless link diversity into account. Considering the the edge nodes are responsible for collecting data from the candidate nodes and sometimes disseminating the update codes or maintenance instructions to the IoT nodes, we need to consider the performance for both collection and dissemination. According to the weights of data collection and dissemination, we devise a comprehensive performance metric $\rho$ for a candidate position $k$ as follows:

$$
\rho_{k}=\alpha p_{k}^{c}+(1-\alpha) p_{k}^{d}
$$

where $p_{k}^{c}$ denotes the performance for data collection and $p_{k}^{d}$ denotes the performance for data dissemination. $p_{k}^{c}$ is given by the sum of all packet delivery ratio (PDR) of one-hop IoT nodes:

$$
p_{k}^{c}=\sum_{i \in N} q_{i k} \times l_{i d x(i, k)}^{i}
$$

where $q_{i k}$ denotes the link quality from node $i$ to node $k$; $i d x(i, k)$ denotes the level index for candidate position $k$ in the range of IoT node $i ; N$ denotes the set of all IoT nodes. Then the physical meaning of $p_{k}^{c}$ is the effective transmission rate for all connected IoT nodes of edge node $k$. The calculation for the dissemination performance $p_{k}^{d}$ is more complex as it involves both link quality and link correlation [16]. We calculate the dissemination performance by combining both link quality and link correlation. Link quality is defined as the packet delivery ratio (PDR) and link correlation is defined for link pairs as follows. Given two links $k \rightarrow i$ and $k \rightarrow j$, the link correlation is calculated as the probability that $k \rightarrow i$ fails a packet given that $k \rightarrow j$ fails the same packet. We denote the above link correlation as $c_{i / j}^{k}$.

Next, we combine link quality and link correlation to calculate the dissemination performance in terms of the expected transmissions required for edge node $k$ to deliver a packet to all target receivers, denoted as $p_{k}^{d}$. $p_{k}^{d}$ can be calculated as follows according to its definition:

$$
p_{k}^{d}=\sum_{m=1}^{+\infty} m P_{n}^{k}(X=m)
$$

where $P_{n}^{k}(X=m)$ denotes that $m$ packet transmissions are required for $k$ to deliver a packet to all $n$ nodes. $P_{n}^{k}(X=m)$ can be calculated as:

$$
P_{n}^{k}(X=m)=P_{n}^{k}(X>m-1)-P_{n}^{k}(X>m)
$$

where $P_{n}^{k}(X>m)$ denotes the number of transmissions for covering $n$ nodes is larger than $m$, which equals to the probability that $m$ transmissions cannot cover all $n$ nodes:

$P_{n}^{k}(X>m)=\left(1-q_{k n}\right)^{m}+P_{n-1}^{k}(X>m)-\left(\left(1-q_{k n}\right) \times c_{N_{n-1} / n}^{k}\right)^{m}$

where $\left(1-q_{k n}\right)^{m}$ denotes the probability that $m$ transmissions cannot cover the $n$-th node, $P_{n-1}^{k}(X>m)$ denotes the probability that $m$ transmissions cannot cover the remaining $n-1$ nodes, i.e., there is at least one node which cannot be covered by $m$ transmissions in the $n-1$ nodes. There is an overlap between the first two items, which is the case that the $n$-th node loses the packet and the $n-1$ nodes are not covered at the same time. Therefore, we need to minus the overlapped probability, i.e., the third item $\left(\left(1-q_{k n}\right) \times c_{N_{n-1} / n}^{k}\right)^{m}$, which is calculated as the probability that $m$ transmissions cannot cover the $n$-th node and at least one node in the remaining $n-1$ nodes is not covered. Please note that " $k$ covers $i$ " here means that node $i$ successfully receives the packet from $k$. 
With Eq.(6), we can calculate $P_{n}^{k}(X>m)$ recursively starting from $P_{1}^{k}(X>m)=\left(1-q_{k 1}\right)^{m}$ as follows:

$$
P_{n}^{k}(X>m)=\sum_{r=1}^{n}\left(\left(1-q_{k r}\right)^{m}-\left(\left(1-q_{k r}\right) \times c_{r-1 / r}^{k}\right)^{m}\right)
$$

Apparently, $c_{0 / 1}^{k}=0$ according to the definition.

Combining Eq.(4)-(7), we can then obtain the performance for data dissemination from an edge node $k$ to all its potential IoT receiver nodes. Then with Eq.(2), we can calculate the utility for each candidate position and sort all candidate positions according to the utility.

Determining the weighing factor in Eq.(2). The $\alpha$ determines weights of collection and dissemination tasks. Suppose there are $s_{c}$ collection traffic and $s_{d}$ dissemination traffic, then we have:

$$
\frac{\alpha}{1-\alpha}=\frac{s_{c}}{s_{d}}
$$

By solving the above equation, $\alpha$ can be obtained:

$$
\alpha=\frac{s_{c}}{s_{c}+s_{d}}
$$

It is worth noting that we assume the offloaded task execution and data processing does not incur noticeable delay for the transmission. Otherwise, the delay incurred by the computational tasks also needs to be considered in the calculation of $\alpha$. The task information $s_{c}$ and $s_{d}$ is provided by the IoT network operator.

Wireless link measurement. As shown in the calculation of the $\rho$ metric, link quality and link correlation need to be measured. We have two alternatives for link measurement: First, link predictions based on the SINR measurements using the model proposed in [17]. By measuring the SINR at all candidate positions, both link quality and link correlation can be predicted. Second, link estimation using the in-packet RSSI sampling. By increasing the sampling rate of the wireless radios, we can extract the byte-wise RSSI readings. For 1 example, we can obtain 127 RSSI samplings from an 127byte packet. As studied in [18], the difference between a given 2 RSSI value and the lowest RSSI value is a good indicator to 3 the error rate for the corresponding byte. Based on the RSSIbased error estimation method [19], we can obtain a series of 4 BER and the variations from the RSSI samplings. Then PDR 5 can be inferred using BER as follows:

$$
q=\prod_{i=1}^{t}\left(1-b_{e}[i]\right)
$$

where $b_{e}[i]$ denotes the estimated BER for $i$-th byte and $t_{9}^{8}$ denotes the packet length in terms of bytes.

Link correlation can be estimated using either single packet ${ }^{10}$ or multiple packets.

1) If single packet is used, the expected link correlation between two links $k \rightarrow i$ and $k \rightarrow j$ is given by:

$$
c_{i / j}^{k}=\sum_{m=1}^{t} \frac{b_{k i}[m] \& b_{k j}[m]}{b_{k j}[m]}
$$

where $b_{k i}[m]$ denotes the binary indicating whether $m$-th byte is erroneous in the packet from $k$ to $i$ and \& denotes the binary AND operation. Specifically, $b_{k i}[m]=1$ if the byte is estimated erroneous.

2) If multiple packets are used, link correlation is calculated as in Eq.(11). The difference is that $b_{k i}[\mathrm{~m}]$ can be directly obtained instead of estimated from the RSSI sampling.

Compared to the measurement with multiple packets, the measurement with single packet can be done more quickly. The drawback is that the accuracy will be decreased. Both measurement schemes can be used according to the application scenario.

\section{E. Deployment algorithm}

Based on the metric $\rho$ proposed in Section III-D, we can sort all the candidate positions. As discussed in Section II, the problem is NP complete. We then propose a heuristic solution by selecting the candidate positions from the position with the best $\rho$. When a candidate position is selected, the IoT nodes within the $m$-th utility level are included as its subscribing receivers. Then we exclude all the covered subscribing receivers, update the $\rho$ values for all candidate positions (expected the chosen positions) and select the position with the highest $\rho$ for deploying the next edge node. The above process continues until all IoT nodes are covered by the edge nodes. The detailed deployment algorithm is described in Alg. 1.

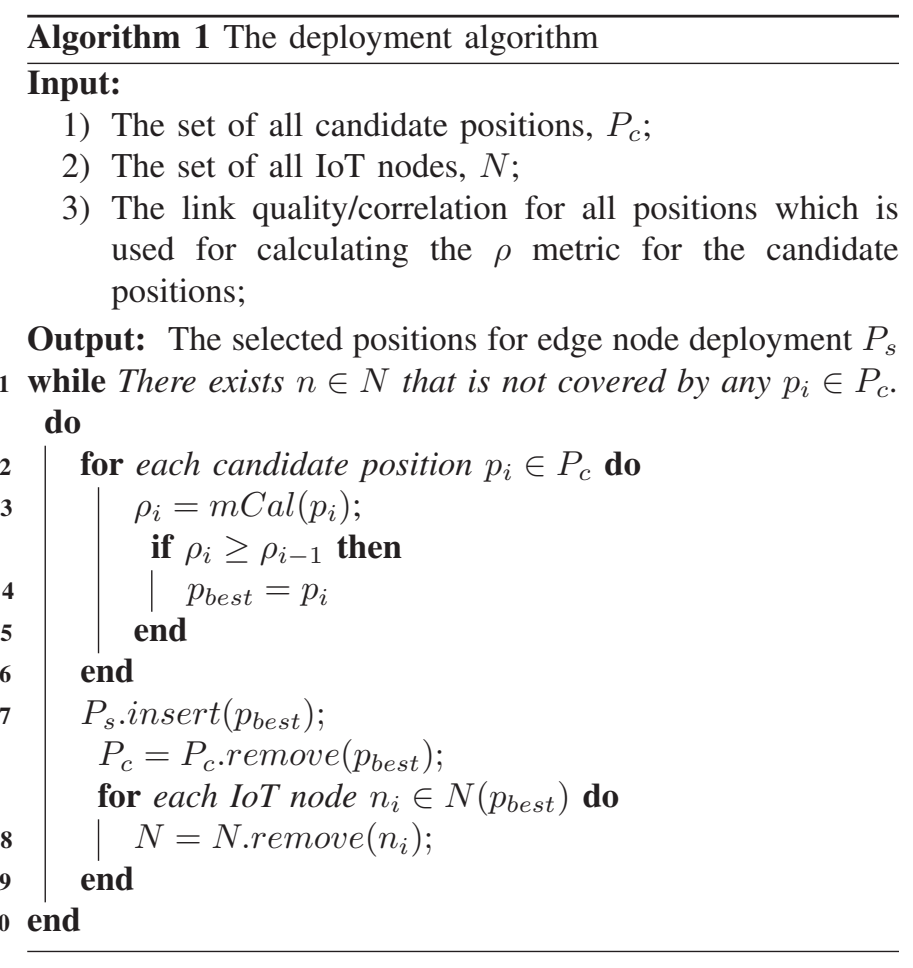

By excluding the selected positions and corresponding IoT nodes, the updated $\rho$ metric still represents the effectiveness of candidate positions. To reduce the computational complexity, we can judge whether the $\rho$ metric is affected before updating. If the previously selected positions have no overlapped IoT nodes with the current position $p_{i}$, then the metric $\rho_{i}$ will remain unchanged for the next round selection. Besides, we can boost the algorithm by selecting the top $\tau$ non-overlapped 
positions in each iteration. With a larger $\tau$, the algorithm runs fast but the selected positions may have worse utilities; With a smaller $\tau$, the algorithm runs slow but the selected positions have better utilities. We will consider using energy efficient clustering algorithms [20], [21], [22] to find the deployment positions in our future work.

\section{Evaluation}

In this section, we evaluate the proposed deployment scheme in comparison with [23], which deploys multiple sinks to a sensor network. The sink nodes are responsible for collecting the sensor data, which play similar role with the edge nodes in our scenario.

\section{A. Experimental settings}

We simulate a 100-node IoT network, where the data traffic in unit time for all IoT nodes are randomly generated. The edge nodes are assumed to be able to receive $T=150 \mathrm{KBps}$. For a given candidate position, the top $m$ IoT nodes with the best $\rho$ are selected to be connected to the position. The sum of the traffic from the selected $m$ nodes does not exceed $T$. Hence $m$ is given by:

$$
m=\max _{n} \sum_{k=0}^{n} r_{k} \leq T
$$

Apparently, $m$ is varying for different positions. The positions of the IoT nodes are randomly placed. We repeat each simulation 100 times to reduce the random variations. We compare the following metrics:

1) The number of edge nodes used to cover all IoT nodes.

2) The performance gain in terms of the average throughput between IoT nodes and the edge nodes.

We tune the following parameters to see in which cases the proposed work performs better or worse.

1) The fraction of high-demand IoT nodes. The highdemand IoT nodes generate five times traffic of the ordinary IoT nodes. We tune the fraction of the highdemand nodes and see the performance gain achieved by the proposed work.

2) The fraction of dissemination tasks. Different IoT networks may have different designing goals, leading to different fractions of dissemination tasks [24]. This fraction has impact on the calculation of $\rho$.

3) The wireless interference. We change the number of interfering wireless APs and compare the performance gains. The interference impacts the wireless diversities, which further impacts the selection of candidate positions.

\section{B. Simulation results}

Figure 4 shows the reduction of deployed edge nodes with different fractions of the high-demand IoT nodes. We change the number of high-demand IoT nodes to see the impact on the reduction. We can see that 1) compared to the existing work based solely on wireless communications, the proposed work always reduces the number of edge nodes. The reason

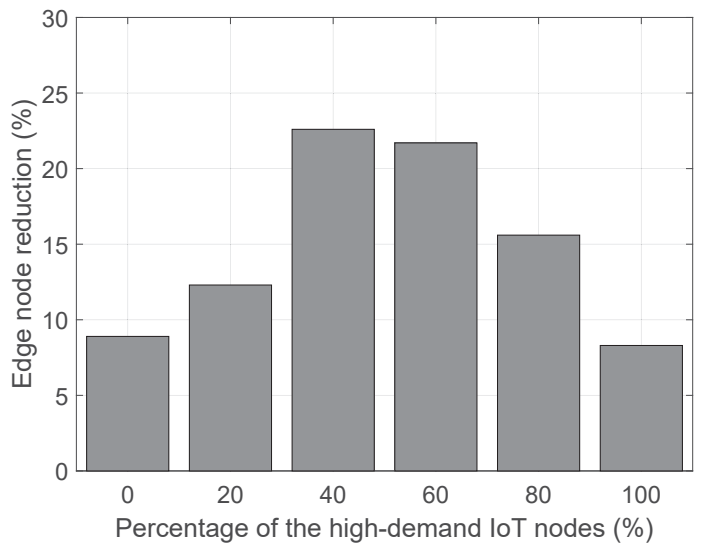

Fig. 4. The reduction on the number of edge nodes with different high-demand IoT nodes.

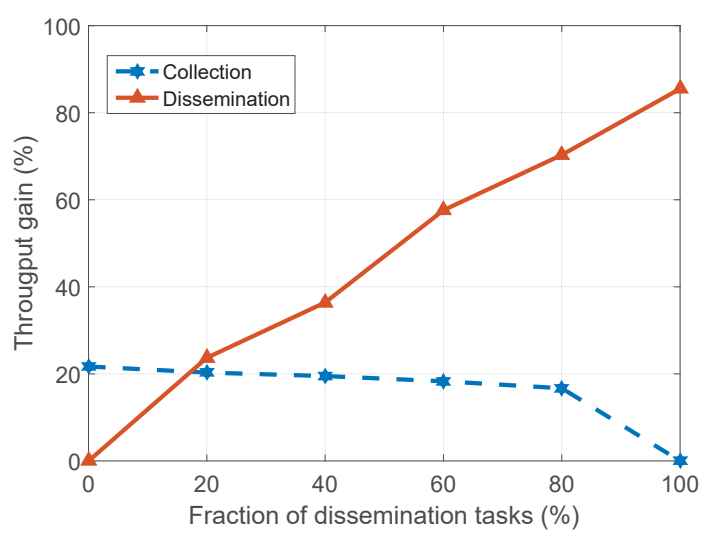

Fig. 5. The throughput gains with varying fractions of dissemination tasks.

is that although link quality is important, the throughput may not be good as high-demand nodes may be assigned to poorquality links. 2) The reduction increases and then decreases, which means our work better suits for the case with more diverse traffic demands from IoT nodes. The reason is that we explicitly consider the traffic diversity in the candidate positions. As a result, when the IoT nodes are more diverse, we have more room for optimization.

Figure 5 depicts the performance gain for both collection tasks and dissemination tasks with varying fraction of the dissemination tasks. We set that the IoT network contains $60 \%$ high-demand nodes. Recall that the dissemination tasks are used for network maintenance or periodic network update. From the results we can see that 1) the performance for data collection is consistently improved. The reason is two-fold. First, wireless link diversity is considered in the proposed work, which reduces the expected packet losses. Second, although the fraction of collection tasks deceases, for each specific collection task, the link diversity is still considered and thus the throughput is improved; 2) the performance for data dissemination significantly increases. This is because the existing works overlook the dissemination task demands. When the fraction of the dissemination tasks increase, the throughput gain increases accordingly. It is also worth noting that in most IoT networks, dissemination is not the dominating 


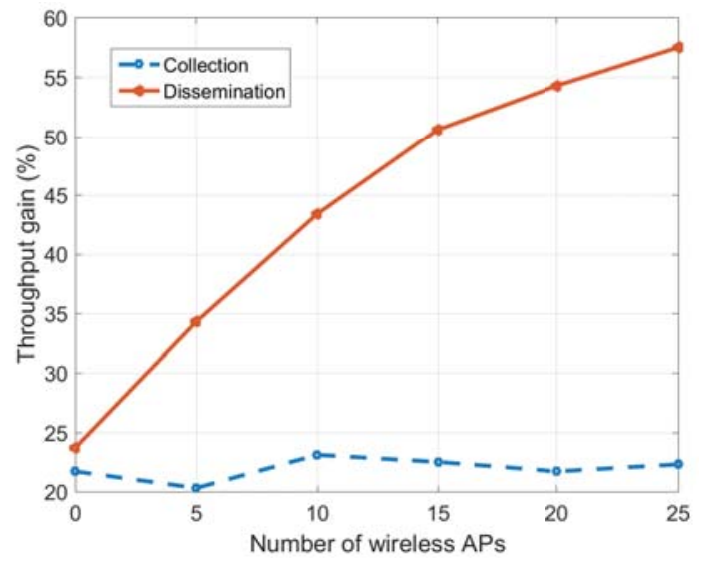

Fig. 6. The throughput gains with varying interfering wireless APs.

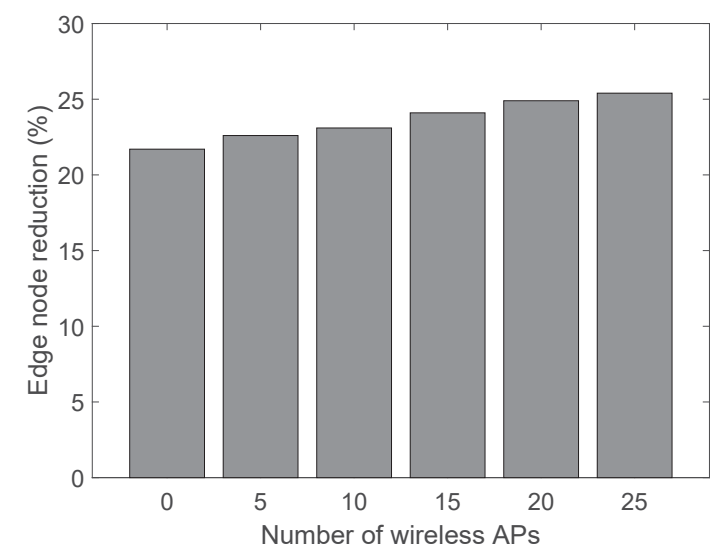

Fig. 7. The reduction on the number of edge nodes with varying interfering wireless APs.

traffic as the main aim is to collect the interesting data from the target area. For some IoT applications with more controlling tasks, the fraction of dissemination tasks should be larger.

Figure 6 shows the throughput gains for collection and dissemination under different number of wireless APs (WiFi). Similar to [25], the impact of WiFi interference is introduced in the simulation by deliberately failing some packet transmissions. The packet losses generated at the sender side will be the correlated packet losses and the packet losses generated at the receiver side will be the independent packet losses. According to the studies in [25], WiFi interference is a dominating reason for correlated packet losses, as a result the packet loss link correlation becomes stronger when WiFi interference becomes stronger. From the results it can be inferred that as the interference becomes stronger, the throughput gain of dissemination becomes larger and the throughput gain of collection remains similar. From the calculation process in Section III-D we can see that link correlation mainly impacts the performance of data dissemination. When link correlation becomes stronger with the interference, there are more optimization space for dissemination.

Figure 7 depicts the reduction of edge nodes with varying number of interfering wireless APs. Different from the experiment in Figure 4, the fraction of high-demand IoT nodes is fixed and the number of interfering nodes is varying. We set $40 \%$ nodes with high traffic demand and $20 \%$ dissemination tasks. It can be inferred that 1) the reduction increases as the interference becomes stronger. From the above analysis on dissemination, we can infer that the increments come from the portion of nodes that have $20 \%$ dissemination tasks. In order to meet the dissemination throughput threshold, more edge nodes will be required for the work without considering dissemination performance. 2) Compared to the results in Figure 4, the reduction changes are much smaller. Therefore, we can conclude that the number of edge nodes is mainly determined by the traffic diversity. The impact of interference on reducing the edge nodes is limited.

\section{RELATED WORKS}

The combination of mobile edge servers and IoT as well as the corresponding benefits are discussed in [26]. Our work differs from the scenario described in [26] in the following ways. First, we consider a large-scale and more practical IoT network, where different IoT nodes are with more diverse demands. Second, we focus on the deployment problem of edge nodes for IoT network while the authors in [26] consider a general idea of combining mobile edge computing and IoT applications.

Although there are few existing works on deploying edge servers for large-scale IoT network, the problem is closely related to the powerful node deployment problem in largescale sensor networks, where the powerful nodes can be either relay nodes or sink nodes [27], [28], [29], [23], [30], [31], [32]. Next, we mainly introduce and discuss the literature for deploying relay nodes or multiple sink nodes in largescale sensor networks. Bredin et al. [28] studied the relay node deployment problem which should meet a survivability requirement. Cheng et al. [29] considers the connectivity constraint in the relay node deployment. Similar to our work, each IoT node is required to be connected to a relay node. Misra et al. [23] additionally consider limiting the candidate positions and propose to select candidate positions before deployment. Our work differs from [23] in that we incorporate the traffic diversity (traffic demand distribution) in the candidate position generation process, therefore providing more reasonable and efficient candidate positions. Nikolov et al. [32] aim at deploying a given number of relays to the network to maximize the communication gains. Bagaa et al. [27] is a recent work that achieves optimal placement of the relays over limited candidate positions. Different from these works, IoT networks contains more diverse nodes and experience more wireless interference. Therefore in our work, we jointly consider the traffic diversity and wireless diversity (especially the link correlation characteristic). As a result, the proposed work is more suitable for large-scale heterogeneous IoT networks and can achieve better throughput gains.

Some works have specific requirements according to the target scenarios. Wu et al. [30] consider the relay node deployment with pipeline inspection. Ma et al. [31] additionally consider the delay constraint for the deployment. Our work is orthogonal to these works, i.e., the above constraints can be 
easily added into our scheme. Besides, the traffic diversity and link correlation are overlooked in these works, which may lead to performance degradation under strong interference scenarios.

\section{CONCLUSION}

In this paper, we propose to deploy edge nodes for realtime data processing in large-scale IoT networks. We identified the key challenges for edge node deployment - the traffic diversity and the wireless diversity. We then propose a novel three-phase deployment approach considering both kinds of diversities. The proposed work aims at minimizing the number of edge nodes and providing real-time processing service for the IoT network. We have conducted simulation experiments and the results show that compared to the existing works that overlooked the two kinds of diversities, the proposed work greatly reduces the number of edge nodes and improves the throughput for both data collection and dissemination.

\section{ACKNOWLEDGMENT}

This work was supported by the National Natural Science Foundation of China (No. 61602095), the National Key Research and Development Program of China (2017YFB1400102), the Fundamental Research Funds for the Central Universities (No. ZYGX2016KYQD098 and No. ZYGX2016J079), National Postdoctoral Program for Innovative Talents of China, and the EU FP7 CLIMBER project under Grant Agreement No. PIRSES-GA-2012-318939.

\section{REFERENCES}

[1] J. Gubbi, R. Buyya, S. Marusic, and M. Palaniswami, "Internet of things (iot): A vision, architectural elements, and future directions," Future generation computer systems, vol. 29, no. 7, pp. 1645-1660, 2013.

[2] W. Du, Z. Xing, M. Li, B. He, L. H. C. Chua, and H. Miao, "Sensor placement and measurement of wind for water quality studies in urban reservoirs," ACM Transactions on Sensor Networks (TOSN), vol. 11, no. 3, p. 41, 2015.

[3] Z. Li, W. Du, Y. Zheng, M. Li, and D. Wu, "From rateless to hopless," IEEE/ACM Transactions on Networking (TON), vol. 25, no. 1, pp. 6982, 2017.

[4] M. Razmara, G. R. Bharati, M. Shahbakhti, S. Paudyal, and R. D. Robinett III, "Bilevel optimization framework for smart building-to-grid systems," IEEE Transactions on Smart Grid, 2016.

[5] A. Bader, H. ElSawy, M. Gharbieh, M.-S. Alouini, A. Adinoyi, and F. Alshaalan, "First mile challenges for large-scale iot," IEEE Coтmunications Magazine, vol. 55, no. 3, pp. 138-144, 2017.

[6] M. Vögler, J. M. Schleicher, C. Inzinger, and S. Dustdar, "A scalable framework for provisioning large-scale iot deployments," ACM Transactions on Internet Technology (TOIT), vol. 16, no. 2, p. 11, 2016.

[7] P. Barnaghi, R. Tönjes, J. Höller, M. Hauswirth, A. Sheth, and P. Anantharam, "Citypulse: Real-time iot stream processing and large-scale data analytics for smart city applications," in Europen Semantic Web Conference (ESWC), vol. 2014, 2014.

[8] F. Chen, Z. Zhao, G. Min, W. Gao, J. Chen, H. Duan, and P. Yang, "Speed control of mobile chargers serving wireless rechargeable networks," Future Generation Computer Systems, vol. 80, pp. 242-249, 2018.

[9] Y. C. Hu, M. Patel, D. Sabella, N. Sprecher, and V. Young, "Mobile edge computinga key technology towards 5g," ETSI White Paper, vol. 11, no. 11, pp. 1-16, 2015.

[10] T. X. Tran, A. Hajisami, P. Pandey, and D. Pompili, "Collaborative mobile edge computing in $5 \mathrm{~g}$ networks: New paradigms, scenarios, and challenges," IEEE Communications Magazine, vol. 55, no. 4, pp. 54-61, 2017.
[11] U. Raza, P. Kulkarni, and M. Sooriyabandara, "Low power wide area networks: An overview," IEEE Communications Surveys \& Tutorials, vol. 19, no. 2, pp. 855-873, 2017.

[12] K. Nair, J. Kulkarni, M. Warde, Z. Dave, V. Rawalgaonkar, G. Gore, and J. Joshi, "Optimizing power consumption in iot based wireless sensor networks using bluetooth low energy," in Green Computing and Internet of Things (ICGCIoT), 2015 International Conference on. IEEE, 2015, pp. 589-593.

[13] Z. Zhao, J. Bu, W. Dong, T. Gu, and X. Xu, "Coco+: Exploiting correlated core for energy efficient dissemination in wireless sensor networks," Ad Hoc Networks, vol. 37, pp. 404-417, 2016.

[14] R. Zhou, Y. Xiong, G. Xing, L. Sun, and J. Ma, "Zifi: wireless lan discovery via zigbee interference signatures," in Proceedings of the sixteenth annual international conference on Mobile computing and networking. ACM, 2010, pp. 49-60.

[15] R. S. Garfinkel, G. L. Nemhauser et al., Integer programming. Wiley New York, 1972, vol. 4.

[16] Z. Zhao, W. Dong, J. Bu, T. Gu, and G. Min, "Accurate and generic sender selection for bulk data dissemination in low-power wireless networks," IEEE/ACM Transactions on Networking (TON), vol. 25, no. 2, pp. 948-959, 2017.

[17] Z. Zhao, W. Dong, G. Guan, J. Bu, T. Gu, and C. Chen, "Modeling link correlation in low-power wireless networks," in Coтриter Соттиnications (INFOCOM), 2015 IEEE Conference on. IEEE, 2015, pp. 990-998.

[18] W. Dong, J. Yu, and P. Zhang, "Exploiting error estimating codes for packet length adaptation in low-power wireless networks," IEEE Transactions on Mobile Computing, vol. 14, no. 8, pp. 1601-1614, 2015.

[19] Z. Zhao, W. Dong, G. Chen, G. Min, T. Gu, and J. Bu, "Embracing corruption burstiness: Fast error recovery for zigbee under wi-fi interference," IEEE Transactions on Mobile Computing, vol. 16, no. 9, pp. 2518-2530, 2017.

[20] L. Zhao, Z. Chen, and Y. Yang, "Parameter-free incremental coclustering for multi-modal data in cyber-physical-social systems," IEEE Access, vol. 5, pp. 21 852-21 861, 2017.

[21] G. Tzortzis and A. Likas, "The minmax k-means clustering algorithm," Pattern Recognition, vol. 47, no. 7, pp. 2505-2516, 2014

[22] L. Zhao, Z. Chen, Z. Yang, Y. Hu, and M. S. Obaidat, "Local similarity imputation based on fast clustering for incomplete data in cyber-physical systems," IEEE Systems Journal, 2016.

[23] S. Misra, S. D. Hong, G. Xue, and J. Tang, "Constrained relay node placement in wireless sensor networks: Formulation and approximations," IEEE/ACM Transactions on Networking (TON), vol. 18, no. 2, pp. $434-447,2010$.

[24] L. Wang and S. Kulkarni, "Gappa: Gossip based multi-channel reprogramming for sensor networks," Distributed Computing in Sensor Systems, pp. 119-134, 2006.

[25] K. Srinivasan, M. Jain, J. I. Choi, T. Azim, E. S. Kim, P. Levis, and B. Krishnamachari, "The $\kappa$ factor: inferring protocol performance using inter-link reception correlation," in Proceedings of the sixteenth annual international conference on Mobile computing and networking. ACM, 2010, pp. 317-328.

[26] P. Corcoran and S. K. Datta, "Mobile-edge computing and the internet of things for consumers: Extending cloud computing and services to the edge of the network," IEEE Consumer Electronics Magazine, vol. 5, no. 4, pp. 73-74, 2016.

[27] M. Bagaa, A. Chelli, D. Djenouri, T. Taleb, I. Balasingham, and K. Kansanen, "Optimal placement of relay nodes over limited positions in wireless sensor networks," IEEE Transactions on Wireless Communications, vol. 16, no. 4, pp. 2205-2219, 2017.

[28] J. L. Bredin, E. D. Demaine, M. T. Hajiaghayi, and D. Rus, "Deploying sensor networks with guaranteed fault tolerance," IEEE/ACM Transactions on Networking (TON), vol. 18, no. 1, pp. 216-228, 2010.

[29] X. Cheng, D.-Z. Du, L. Wang, and B. Xu, "Relay sensor placement in wireless sensor networks," Wireless Networks, vol. 14, no. 3, pp. 347 $355,2008$.

[30] D. Wu, D. Chatzigeorgiou, K. Youcef-Toumi, S. Mekid, and R. BenMansour, "Channel-aware relay node placement in wireless sensor networks for pipeline inspection," IEEE Transactions on Wireless Communications, vol. 13, no. 7, pp. 3510-3523, 2014.

[31] C. Ma, W. Liang, and M. Zheng, "Set-covering-based algorithm for delay constrained relay node placement in wireless sensor networks," in Communications (ICC), 2016 IEEE International Conference on. IEEE, 2016, pp. 1-6.

[32] M. Nikolov and Z. J. Haas, "Relay placement in wireless networks: Minimizing communication cost," IEEE Transactions on Wireless Communications, vol. 15, no. 5, pp. 3587-3602, 2016. 